\title{
INTEGRALS WITH NONDEGENERATE CRITICAL POINTS
}

\author{
BY K. UHLENBECK
}

Communicated by Richard Palais, June 23, 1969

Palais [3] and Smale [6] have given conditions under which a function on a Hilbert manifold is a Morse function. Palais [4] and the author [7] have given classes of integrals on manifolds of sections of fiber bundles which satisfy the condition (C) of Palais and Smale and therefore satisfy a Ljusternik-Schnirelmann category theory [4], but for Morse theory the critical points must in addition be nondegenerate. We now outline a proof of a conjecture of Palais': that for almost all choices of Dirichlet boundary values, certain of these integral have only nondegenerate critical points on that boundary value manifold and therefore satisfy the full Morse theory. The model for these results and immediate corollary is the fact that the conjugate points for the geodesic problem on a Riemannian manifold are residual. For any two Riemannian manifolds $M$ and $N$ with $\partial M \neq \varnothing, \partial N=\varnothing$, and $2 k>\operatorname{dim} M$, we construct a canonical function $E_{k}$ on $H_{k}(M, N)$ which is a Morse function on $H_{k}(M, N)_{\lambda}$ for almost all $\lambda$.

We first prove an abstract theorem. Let $\pi: H \rightarrow G$ be a separable $C^{\infty}$ fiber bundle over a Banach manifold $G$ with a Banach manifold as fiber and identify $\pi^{*} T^{*}(G)$ with $(d \pi)^{*} T^{*}(G) \subseteq T^{*}(H) . H_{g}=\pi^{-1}(g)$ for $g \in G$.

Theorem 1. Let $J$ be a $C^{2}$ function on $H$ and consider the $C^{1}$ function $d J: H \rightarrow T^{*}(H)$. If

(1.1) The Hessian of $J \mid H_{0}$ is a Fredholm operator at all critical points for all $g \in G$, and

(1.2) $d J \pitchfork \pi^{*} T^{*}(G)$

then the subset $\left\{g \in G|J| H_{0}\right.$ has only nondegenerate critical points $\}$ is residual in $G$.

The proof of Theorem 1 follows directly from a theorem on transversality similar to the theorem given by Abraham in [1]. Since (1.2) is hard to verify directly, we state an equivalent condition (1.3).

(1.3) At critical points of $J \mid H_{0}$ for all $g \in G$ we have

$$
d^{2} J_{s}: T_{s}(H) \rightarrow T_{s}\left(T^{*}(H)\right) \cong T_{s}^{*}(H) \oplus T_{s}(H) \stackrel{\left(d i_{\theta}\right)^{*}}{\longrightarrow} T_{s}^{*}\left(H_{\theta}\right)
$$

where $i_{g}: H_{g} \subseteq H$, and we require $\left(d i_{o}\right)^{*} \circ d^{2} J_{s}$ to be onto.

We use the definitions and terminology of Palais [5]. Let $p: E \rightarrow M$ be a smooth finite-dimensional fiber bundle over a compact $n$ 
manifold $M$ with boundary $\partial M$ and a smooth measure $u$. Let $J(s)$ $=\int_{M} L\left(j^{k} s(x)\right) d u(x)$ where the Lagrangian $L$ is at least a $C^{3}$ function on the $k$-jet bundle of $E$. If $Q(E)$ is a function manifold, we denote the manifold of functions with the derivatives of $\lambda$ up through order $k-1$ on the boundary by $Q(E)_{\lambda}$. Similarly, if $B$ is defined on $Q(E)$, $B_{\lambda}=B \mid Q(E)_{\lambda}$. We also use the terminology that a linear elliptic system $B$ of order $2 k$ between two vector bundles $\eta$ and $\xi$ over $M$ has the unique continuation property if the formal adjoint operator $B^{*}: H_{k}\left(\xi^{*}\right) \rightarrow H_{-k}\left(\eta^{*}\right)$ is onto. When the coefficients of $B$ are smooth enough, this is equivalent to the usual interpretation that $B$ has no functions in its kernel which are extendable smoothly by 0 across $\partial M$.

Theorem 2. Let $J$ and $E$ be as above, and assume $L$ is of order $k>n / 2$ and weight $2 k$, so $J: H_{k}(E) \rightarrow R$ is $C^{\infty}$. Assume that for all $\lambda \in C^{k+r}(E)$,

(2.1) the second variation of $J_{\lambda}$ can be interpreted as a linear elliptic system of order $2 k$, and

(2.2) at critical points $s$ of $J_{\lambda}$, this elliptic system has the unique continuation property.

Then the subset $\left\{\lambda \in C^{k+r}(E) \mid J_{\lambda}\right.$ has only nondegenerate critical points $\}$ is residual in $C^{k+r}(E)$.

Corollary. $C^{k+r}(E)$ can be replaced by $C^{\infty}(E)$.

Theorem 2 is proved by letting $H=\left\{s \in H_{k}(E) \mid s\right.$ has $C^{k+r}$ Dirichlet boundary values $\}$ and $G=$ space of $C^{k+r}$ boundary values. The corollary follows from the openness of transversality. The proofs of Theorems 1 and 2 can be modified to give Theorems 3 and 4 . It may be desirable to replace the spaces $C^{2 k+\alpha}$ and $C^{k+\alpha}$ by other separable function spaces.

TheOREM 3. Let $\xi$ and $\eta$ be vector bundles over $M$ and $B$ a nonlinear elliptic system of order $2 k$ from $\xi$ to $\eta$. If $B: C^{2 k+\alpha}(\xi) \rightarrow C^{\alpha}(\eta)$ is $C^{r}$, $r>$ maximum (index $B, 0)$, and $\left(d B_{s}\right)^{*}$ has the unique continuation property for $s \in B^{-1}(0)$ with $C^{2 k+R}$ boundary values, then $\left\{\lambda \in C^{2 k+R}(\xi) \mid B_{\lambda}\right.$ has zero as a regular value $\}$ is residual in $C^{2 k+R}(\xi)$.

Theorem 4. Let $J(s)=\int_{M} L\left(j^{k} s(x)\right) d u(x)$, where $L$ is at least $C^{3}$. Assume that for all $\lambda \in C^{k+r}(E),(2.1)$ and (2.2) are valid. Then $J$ is a $C^{2}$ function of $C^{k+\alpha}(E)$ and $\left\{\lambda \in C^{k+r}(E) \mid\right.$ at critical points of $J_{\lambda}$ the Hessian $d^{2} J_{\lambda, 8}$ extends to a nondegenerate bilinear form on $\left.H_{k}\left(s^{*} T F E\right)_{0}\right\}$ is residual in $C^{k+r}(E)$.

In view of Theorem 4 , we suggest the following definition: A criti- 
cal point $s$ of an integral defined on $L_{k}^{p}(E)_{\lambda}(p>2)$ is said to be nondegenerate if $s \in C^{k+\alpha}(E)$ and if $d^{2} J_{s}$ extends to a nondegenerate bilinear form on $H_{k}\left(s^{*} T F E\right)_{0}$.

The main difficulty in the application of these results is in giving conditions on an elliptic system with nonanalytic coefficients to insure the unique continuation property. The estimates of Hörmander [2] show that an elliptic system $A$ has the unique continuation property across any surface, if either:

(5.1) the symbol of $A$ is a scalar, and $\sigma_{x} A(z+t v)=0$ has simple complex roots in $t$ for all $z, v \in T_{x}(M), z \neq c v$. The coefficients of $A$ must be $C^{\mathbf{1}}$.

(5.2) $A=A_{k}, A_{1}=B_{1}$ and either $A_{i}=A_{i-1} B_{i}+Q_{i}$ or $A_{i}=B_{i} A_{i-1}+Q_{i}$ where the $B_{i}$ satisfy (5.1), order $Q_{i}<\operatorname{order} A_{i}-i / 2$, and the coefficients are all $C^{q}$ for $q>$ order $A+1$.

In our applications, let $E=M \times N$, for $M$ and $N$ finite dimensional Riemannian manifolds, $\partial M \neq \varnothing$ and $\partial N=\varnothing$. For the geodesic problem, we take $M=[0,1]$ and define

$$
J(s)=\int_{0}^{1}|D s|^{2} d t
$$

which is a $C^{\infty}$ function on $H_{k}([0,1], M)$. By using Theorem 2 we find that the set of $(p, q) \in N \times N$ such that $\left\{J \mid H_{1}([0,1], M)_{(p, q)}\right.$ has nondegenerate critical points (equivalently, $p$ and $q$ are nonconjugate points) $\}$ is residual in $N \times N$.

For arbitrary $M$ we can construct the integral

$$
J(s)=\int_{M}|D s|^{2} d u
$$

on $C^{1}(M, N)$, since

$$
D s(x) \in L\left(T_{x}(M), T_{s(x)}(N)\right) \cong T_{x}^{*}(M) \otimes T_{s(x)}(N)
$$

which has a natural metric on it. Theorem $4(k=1)$ can be applied, since the second variation satisfies (5.1).

As in the second example, we can consider $D s$ as a section of the bundle $T^{*}(M) \otimes s^{*} T(N)$ when $s: M \rightarrow N$ is a smooth map. This bundle has a natural metric and covariant differential, which can be iterated to give

$$
E_{k}(s)=\int_{M} \sum_{j=0}^{k-1}\left|\nabla^{j} D s\right|^{2} d u .
$$

When $k>\operatorname{dim} M / 2$, Theorem 2 can be applied with $r>k+1$, since the critical points are differentiable enough, and the second variation 
factors as in (5.2). Since $E_{k}$ also satisfies condition (C) on $H_{k}(M, N)$ [7], we get the final theorem.

THEOREM 6. For $2 k>$ dimension $M$ and almost all boundary values $\lambda \in C^{2 k+r}(M, N), 1<r \leqq \infty, E_{k}$ is a $C^{\infty}$ Morse function on $H_{k}(M, N)_{\lambda}$.

\section{REFERENCES}

1. R. Abraham and J. Robbin, Transversal mappings and flows, Benjamin, New York, 1967.

2. L. Hörmander, Linear partial differential operators, Chapter 8, Academic Press, New York and Springer-Verlag, Berlin, 1963. MR 28 \#4221.

3. R. Palais, Morse theory on Hilbert manifolds, Topology 2 (1963), 299-340. MR 28 \#1633.

4. - Ljusternik-Schnirelmann theory on Banach manifolds, Topology 5 (1966), $115-132$

5. - Foundations of global non-linear analysis, Benjamin, New York, 1968.

6. S. Smale, Morse theory and a non-linear generalization of the Dirichlet problem, Ann. of Math. (2) 80 (1964), 382-396. MR 29 \# 2820.

7. K. Uhlenbeck, Thesis, Brandeis University, Waltham, Mass., 1968.

Massachusetts Institute of Technology, Cambridge, Massachusetts 02139 